\title{
Nanoscale
}

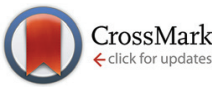

Cite this: Nanoscale, 2015, 7, 19432

Received 25th May 2015,

Accepted 23rd October 2015

DOI: $10.1039 / c 5 n r 03444 b$

www.rsc.org/nanoscale

\section{How fast is the reaction of hydrated electrons with graphene oxide in aqueous dispersions? $\uparrow$}

\author{
Axel Kahnt, ${ }^{* a}$ Roman Flyunt, ${ }^{\mathrm{b}}$ Christian Laube, ${ }^{\mathrm{b}}$ Wolfgang Knolle, ${ }^{\mathrm{b}}$ Siegfried Eigler, \\ Ralf Hermann, ${ }^{d}$ Sergej Naumov ${ }^{\mathrm{b}}$ and Bernd Abel ${ }^{{ }^{\mathrm{b}}, \mathrm{d}}$
}

\begin{abstract}
Understanding the mechanism of the reduction of graphene oxide (GO) is a key-question in graphene related materials science. Here, we investigate the kinetics of the reaction of radiolytically generated hydrated electrons with GO in water. The electron transfer proceeds on the ns time scale and not on the ps time scale, as recently reported by Gengler et al. (Nat. Commun., 2013, 4, 2560).
\end{abstract}

\section{Introduction}

Carbon based nanomaterials with dimensionalities 0D (fullerenes), 1D (carbon nanotubes) and 2D (graphene/graphene oxide) have gained large scientific interest in the past decade. ${ }^{1,2}$ In recent years a particular focus has been set on 2D carbon materials, such as graphene and graphene oxide (GO). These materials are, however, correlated with a number of practical issues. In particular, the production of high quality monolayer graphene with the perspective of large scale applications/ productions, is still challenging. ${ }^{3,4}$ For graphene oxide (GO) on the other hand up-scalable synthetic procedures starting from graphite are established. ${ }^{4}$ Moreover in aqueous dispersions, GO occurs - even without surfactants - as a monolayer material. The major drawback of GO is, that the quality of the carbon framework depends strongly on the preparation conditions. Therefore GO prepared according to common pro-

\footnotetext{
${ }^{a}$ Department of Chemistry and Pharmacy \& Interdisciplinary Center for Molecular Materials, Chair of Physical Chemistry I, Friedrich-Alexander-Universitat ErlangenNürnberg, Egerlandstrasse 3, 91058 Erlangen, Germany.E-mail: axel.kahnt@fau.de ${ }^{b}$ Leibniz Institute of Surface Modification (IOM), Chemical Department, Permoserstr. 15, 04318 Leipzig, Germany. E-mail: bernd.abel@iom-leipzig.de

${ }^{c}$ Department of Chemistry and Pharmacy, Institute of Advanced Materials and Processes (ZMP), Friedrich-Alexander-Universität Erlangen-Nürnberg (FAU), Henkestr. 42, 91054 Erlangen and Dr.-Mack Str. 81, 90762 Fürth, Germany ${ }^{d}$ Wilhelm-Ostwald-Institute for Physical and Theoretical Chemistry, Faculty of Chemistry and Mineralogy, University of Leipzig, Permoserstr. 15, 04318 Leipzig, Germany

$\dagger$ Electronic supplementary information (ESI) available: Absorption spectrum of $\mathrm{GO}$, pulse radiolysis and laser photolysis transient absorption spectra, kinetic simulation, frontier molecular orbitals, and possible reaction pathways induced by photoexcitation. See DOI: $10.1039 / \mathrm{c} 5 \mathrm{n} r 03444 \mathrm{~b}$
}

cedures displays a highly defective $\mathrm{sp}^{2}$ carbon framework with edges of defects functionalized with oxygen containing groups such as $-\mathrm{COOH},-\mathrm{OH}, \mathrm{C}=\mathrm{O}$, etc. ${ }^{4}$ Bearing this in mind, the reduction of GO to graphene is a smart route toward graphene with various defect densities. In this regard countless methods for the reduction of GO have been published in recent years - in particular "classical" preparative approaches. The results obtained from reductants such as hydrazine, dimethylhydrazine, hydroquinone, $\mathrm{NaBH}_{4}, \mathrm{TiO}_{2}, \mathrm{HI}$ and others have been recently summarized. ${ }^{4-6}$ In comparison with the above methods, much less work was reported applying e.g. photo- or radiation chemical methods as preparative chemical methods. Recently we investigated the reduction of three different graphene oxides and the properties of the formed rGO (reduced graphene oxide) utilizing hydroxylalkyl radicals derived from alcohols and solvated electrons as reducing agents. The properties of the formed rGOs were analyzed by steady state electron beam radiolysis, ${ }^{7,8}$ and a better efficiency of this method was found compared to preparative approaches. But up to now, detailed mechanistic and kinetic aspects of these reactions remained elusive. Even for the "classical" preparative reduction, mechanistic and kinetic studies are only spotted in the literature and novel knowledge about the mechanism of reduction is highly desired. ${ }^{9,10}$

Although each of the reduction methods has its own advantages, light-induced reduction of GO appears to be a smart and interesting way of producing reduced GO (rGO) while avoiding the use of harsh chemicals. The photochemical reduction of GO was studied altering the wavelength, exposure time or light intensity, both in solids or in the liquid phase. ${ }^{11-15}$ Although these great efforts have been undertaken the key-questions of understanding the dynamics and details of the reduction mechanism remain largely unanswered yet. In the present contribution we want to shed light on the issue of how GO is photo-reduced, what the reducing species is, and what the role of the solvated electron in this process is. In order to understand photo-reduction of graphene and the role of solvated electrons in this process we generate solvated electrons via radiation chemical methods and study the kinetics under 
very well defined experimental conditions relevant for photoreduction, as outlined below.

Recently, Gengler et al. ${ }^{1}$ investigated the nature of the ultraviolet photo-reduction of GO in water by using femtosecond laser pulses in a pump-probe experiment. A water dispersion of GO was irradiated by an ultraviolet femtosecond pump pulse, followed by a second femtosecond visible probe pulse. By varying the delay between the pump and the probe pulses the evolution of the reaction was monitored in real time. Their results appear to support a recently proposed model for GO photo-reduction in water: the ultraviolet light $(4.6 \mathrm{eV})$ is absorbed by the solvent through a nonlinear process, namely two-photon absorption. This process excites the water above its photoionization threshold $(6.5 \mathrm{eV})$ leading to the generation of solvated electrons, which are then responsible for the production of rGO. The entire reduction dynamics are unveiled by transient absorption measurements and are found to occur on a picosecond time scale. Here, we present a kinetic study on the defined electron pulse radiolysis reduction of three GO samples in aqueous dispersion (in order to display the variability of kinetics for different materials and material qualities). We employed single-layer GO purchased from Cheaptubes.com (CT-GO) and from Nanoinnova Technologies (NI-GO) as well as home-made GO with an almost intact carbon framework $\left(\right.$ ai-GO) ${ }^{16}$ (for details see the Experimental section). By applying this technique (electron pulse radiolysis) we exclusively generate 'truly' solvated electrons $\mathrm{e}_{(\mathrm{aq})}{ }^{-}$and explicitly exclude excitation of GO leading to the decomposition of GO. The new experiments aim at shedding some more light on the question of the nature of the reducing species and the mechanism in the photo-reduction of graphene oxide (GO) with intense pulsed UV light.

\section{Experimental section}

\section{Materials}

All chemicals were purchased from commercial sources in highest purity and used as received. Three different GO samples have been investigated in this work: (I) single-layered GO purchased from Cheaptubes.com (USA) - further called CT-GO, (II) GO from Nanoinnova Technologies (Madrid, Spain) - further called NI-GO, and (III) home-made GO with an almost intact carbon framework - further called ai-GO synthesized according to Eigler et al. ${ }^{16}$ Dispersions of GO were prepared in Millipore water using bath ultrasonication, for details see ref. 7 .

\section{Pulse radiolysis}

The samples were saturated with $\mathrm{N}_{2}$ and irradiated with high energy electron pulses ( $1 \mathrm{MeV}, 15 \mathrm{~ns}$ duration) by using a pulse transformer type electron accelerator (Elit - Institute of Nuclear Physics, Novosibirsk, Russia). The dose delivered per pulse was measured by electron dosimetry. ${ }^{17}$ Doses between and 2.3 and 100 Gy were employed. The optical detection of the transients was carried out with a detection system consist- ing of a pulsed (pulser MSP 05 - Müller Elektronik Optik) xenon lamp (XBO 450, Osram), a SpectraPro 500 monochromator (Acton Research Corporation), a R 9220 photomultiplier (Hamamatsu Photonics), and a $500 \mathrm{MHz}$ digitizing oscilloscope (TDS 640, Tektronix).

\section{Computational methods}

Quantum chemical calculations were performed using the Density Functional Theory (DFT) employing the B3LYP ${ }^{18,19}$ functional as implemented in the Jaguar program. ${ }^{20}$ The structures of the studied molecules were optimized in water using the Jaguar's Poisson-Boltzmann solver $(\mathrm{PBF})^{21}$ at the B3LYP/ 6-31(d)/PBF level of theory. The frequency analysis was performed to characterize the stationary points on the potential surface and to obtain total enthalpy $(H)$ and Gibbs free energy $(G)$ at $298 \mathrm{~K}$. The reaction enthalpies $(\Delta H)$ and Gibbs free energies of reaction $(\Delta G)$ were calculated as the difference of the calculated $H$ and $G$ between the reactants and products respectively. The calculations were performed on the model systems of GO with the hexagonal $5 \times 5$ graphene sheet and a $\mathrm{COO}^{-}$or $\mathrm{COOH}$ group connected to the zig-zag edges, which as expected displays higher reactivity as compared to the armchair edges, as well as on a $5 \times 8$ graphene sheet with an epoxy functionality. The extensive calculations on the possible reaction pathways induced by photo-excitation $(266 \mathrm{~nm})$, which could lead to reduced graphene oxide (rGO), are given in the ESI (Fig. S7 and S8 in the ESI†).

\section{fs-Laser photolysis experiments}

The femtosecond transient absorption measurements were carried out with a CPA-2101 femtosecond laser (Clark MXR). The excitation wavelength $(256 \mathrm{~nm})$ was created by third harmonic generation. Laser output energies between 100 and $400 \mathrm{~nJ}$ per pulse were selected.

\section{Results}

With pulse radiolysis the nature and yields of the generated primary species in the system can be controlled with high precision. Indeed, three highly reactive species, namely ${ }^{\circ} \mathrm{H},{ }^{\circ} \mathrm{OH}$, and $\mathrm{e}_{(\mathrm{aq})}{ }^{-}$(eqn (1)), besides the molecular products $\mathrm{H}_{2}$ and $\mathrm{H}_{2} \mathrm{O}_{2}$ are formed due to water radiolysis. ${ }^{22}$ Under the conditions employed ( $\mathrm{N}_{2}$-saturated, aqueous solutions at natural $\mathrm{pH}$ containing $5 \mathrm{vol} \% \mathrm{t}$-butanol) only hydrated electrons remain available for the reaction with $\mathrm{GO}$, since ${ }^{\circ} \mathrm{H}$ and ${ }^{\circ} \mathrm{OH}$, are completely scavenged by $t$-butanol via hydrogen abstraction (eqn (2)). The here formed ${ }^{\circ} \mathrm{CH}_{2} \mathrm{C}\left(\mathrm{CH}_{3}\right)_{2}(\mathrm{OH})$ radicals (eqn (2)) are known as redox inert species of low reactivity ${ }^{23}$ and hence any kind of their possible reaction with GO can be neglected.

The transient absorption spectra, obtained upon electron pulse radiolysis of aqueous, $\mathrm{N}_{2}$-saturated ai-GO dispersion containing 5 vol\% $t$-butanol after different time delays are shown in Fig. 1a. All of them correspond to the spectrum of the hydrated electron, ${ }^{24}$ which disappear almost completely $5 \mu \mathrm{s}$ after the electron pulse. The decay of $\mathrm{e}_{(\mathrm{aq})}{ }^{-}$at different GO con- 

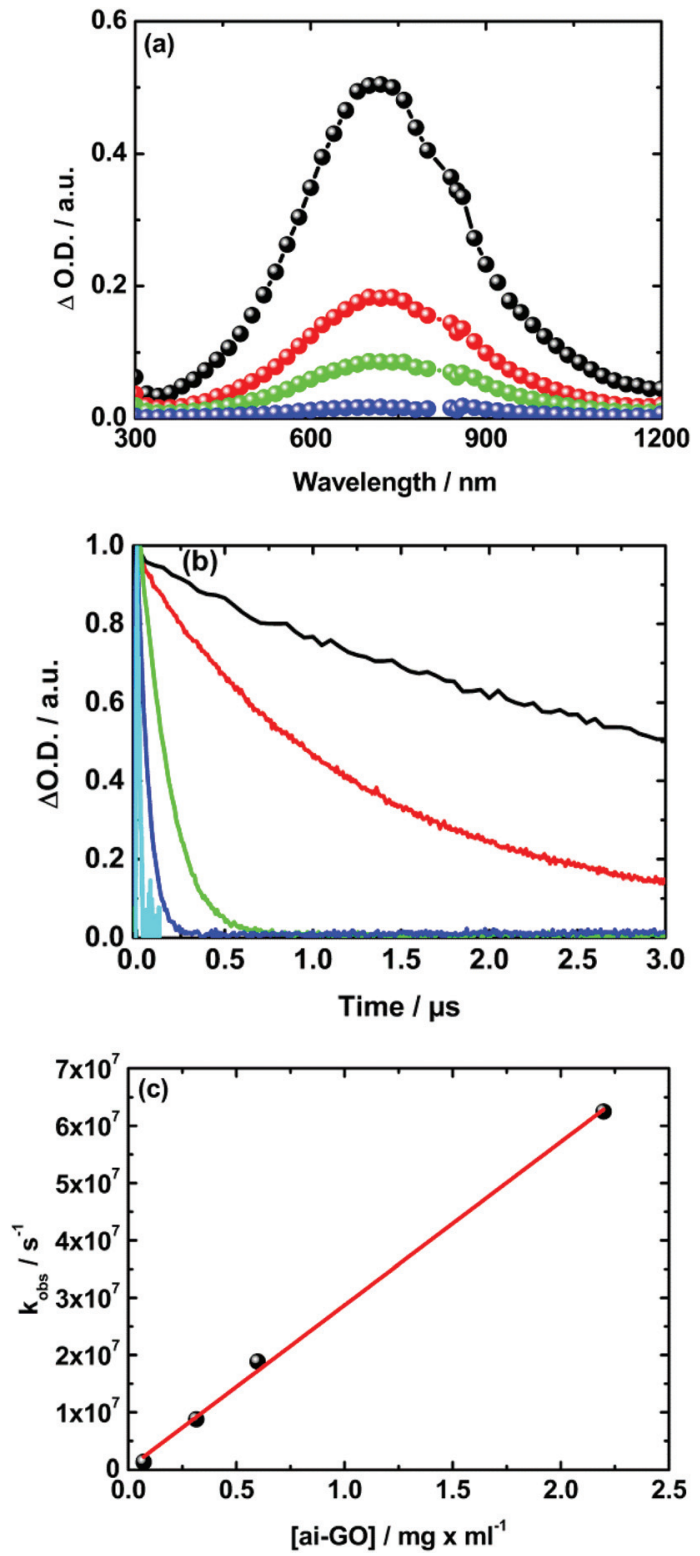

Fig. 1 (a) Transient absorption spectra obtained upon electron pulse radiolysis (100 Gy, $15 \mathrm{~ns}$ FWHM) of $0.07 \mathrm{mg} \mathrm{ml}^{-1}$ ai-GO in $\mathrm{N}_{2}$-saturated aqueous solution in the presence of $5 \mathrm{vol} \% t$-butanol with time delays of 50 ns (black), $1 \mu \mathrm{s}$ (red), $2 \mu \mathrm{s}$ (green) and $5 \mu \mathrm{s}$ (blue) after the electron pulse. (b) Corresponding absorption time profiles at $720 \mathrm{~nm}$ for solutions containing no ai-GO (black curve), $0.07 \mathrm{mg} \mathrm{ml}^{-1}$ ai-GO (red curve), $0.31 \mathrm{mg} \mathrm{ml}^{-1}$ ai-GO (green curve), $0.60 \mathrm{mg} \mathrm{ml}^{-1}$ ai-GO (blue curve) and $2.20 \mathrm{mg} \mathrm{ml}^{-1}$ ai-GO (cyan curve). (c) Plot of the pseudo-first-order rate constants vs. the ai-GO concentration for the decay of the hydrated electrons measured at $720 \mathrm{~nm}$.

centrations has been followed at $720 \mathrm{~nm}$ as illustrated in Fig. 1b. The signals disappear following a pseudo-first-order kinetics (note: the concentration of solvated electrons is much lower than the graphene flake 'concentration') and the corresponding linear dependence of $k_{\text {obs }} v s$. ai-GO concentration is plotted in Fig. 1c. Typical fits are displayed in the appendix (ESI) in Fig. S1. $\dagger$

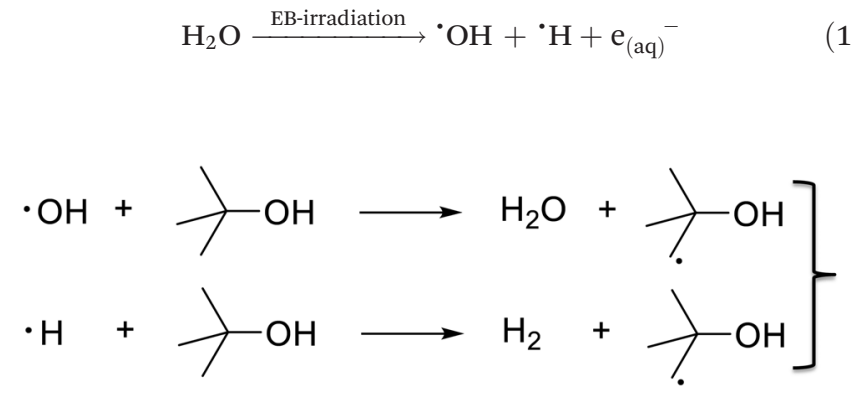

(2)

For the other GO samples (NI-GO and CT-GO) we found a similar behaviour, i.e., transient absorption spectra dominated by the absorptions of $\mathrm{e}_{(\mathrm{aq})}{ }^{-}$(Fig. S2a and S3a, ESI $\dagger$ ). The rate constants were determined from the decay of the transient absorption of $\mathrm{e}_{(\mathrm{aq})}{ }^{-}$at $720 \mathrm{~nm}$ (Fig. S2b and $\mathrm{S} 3 \mathrm{~b} \dagger$ ). From the slope of the linear fit of the pseudo-first-order rate constants $v s$. the GO concentration (Fig. S2c and S3c $\dagger$ ) the bimolecular rate constants for the reaction of the particular GO with $\mathrm{e}_{(\mathrm{aq})}{ }^{-}$ were obtained and are collected in Table 1, displaying the variability of the different GO samples. Reduction of the GO has been controlled routinely via XPS (core level photoelectron spectroscopy), UV-VIS spectroscopy, and conductivity measurements such as shown in ref. 7. For a proof that reduction is actually taking place under EB radiation conditions we refer to ref. 7 and 8.

Since for measurements resulting from high absorbed doses, i.e., $100 \mathrm{~Gy}$, the pseudo-first order approximation may provide rate constants that appear somewhat higher than expected because it neglects second order effects resulting from the recombination of $\mathrm{e}_{(\mathrm{aq})}{ }^{-}$. In order to estimate any error in this direction we performed kinetic simulations using ACUCHEM $^{25}$ for the $720 \mathrm{~nm}$ time profiles (see e.g., Fig. S4 in the ESI $\dagger)$. From these simulations a rate constant of $(2.65 \pm$ $0.20) \times 10^{7} \mathrm{ml} \mathrm{mg}^{-1} \mathrm{~s}^{-1}$ was obtained for the reaction of $\mathrm{e}_{(\mathrm{aq})}{ }^{-}$ with ai-GO, indicating that under the applied conditions the pseudo-first order approximation leads to reasonable results and very small errors. Please note, GO is a label for a number of different heterogeneous materials (with very different defect numbers and features, i.e., functional groups), for which no classical solution concentration of the reactant in terms of mol $\mathrm{l}^{-1}$ can be given. Instead we give rate constants (in

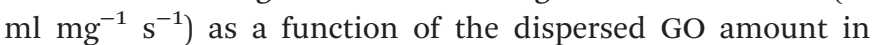
solution $\left(\mathrm{mg} \mathrm{ml}^{-1}\right)$. Note, the concentration of GO varies somewhat for the different samples because of the different solubilities. The concentration of GO is nevertheless still much higher compared to the $\mathrm{e}_{(\mathrm{aq})}{ }^{-}$concentration so that the conditions are still justifying the pseudo first order approximation.

Table 1 Rate constants (in $\mathrm{ml} \mathrm{mg}^{-1} \mathrm{~s}^{-1}$ ) for the reactions between the $\mathrm{e}_{(\mathrm{aq})}{ }^{-}$and three different $\mathrm{GO}$ samples

\begin{tabular}{llll}
\hline Species & ai-GO & CT-GO & NI-GO \\
\hline $\mathrm{e}_{(\mathrm{aq})}{ }^{-}$ & $(2.8 \pm 0.2) \times 10^{7}$ & $(1.2 \pm 0.1) \times 10^{7}$ & $(2.3 \pm 0.3) \times 10^{7}$
\end{tabular}


(a)
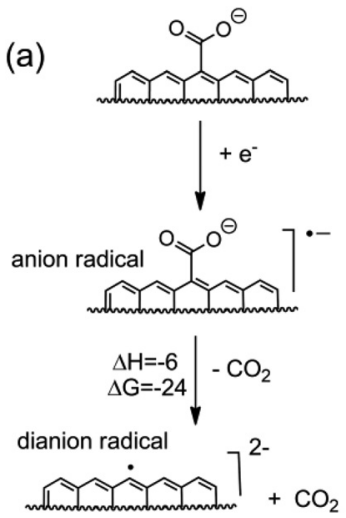

Fig. 2 Calculated energetics of the possible reaction pathways of $\mathrm{CO}_{2}$ scission after the interaction of the $\mathrm{GO}$ with $\mathrm{e}_{(\mathrm{aq})}{ }^{-}$: (a) with $\mathrm{COO}^{-}$and (b) with $\mathrm{COOH}$ groups, leading to the formation of the corresponding $\mathrm{GO}$ radical anion and shows its further stabilization by decarboxylation. $\Delta H$ and $\Delta G$ are given in $\mathrm{kcal} \mathrm{mol}^{-1}$. Here for the case (a) after addition of the electron, we should have dianion radical species immediately, which remains as dianion radical species after loss of carbon dioxide.

The goal of further investigations was to study the reduction of $\mathrm{GO}$ by $\mathrm{e}_{(\mathrm{aq})}{ }^{-}$with quantum chemical calculations. Here two model GO systems, namely $5 \times 5$ and $8 \times 5$, were studied; both for $\mathrm{GO}$ containing a $\mathrm{COO}^{-}$or a $\mathrm{COOH}$ group and $\mathrm{GO}$ bearing an epoxy group, which is hydrolysed and easily transformed to two $\mathrm{OH}$ groups. Because the calculated thermochemical parameters such as $\Delta H$ and $\Delta G$ were very similar, only one model is shown here in Fig. 2 and 3, respectively.

The investigations revealed for both the tested models a high driving force for the decarboxylation of the $\mathrm{COOH}$ and $\mathrm{COO}^{-}$group and the removal of the $\mathrm{OH}$ groups respectively. As a result of these quantum chemical calculations, it is reasonable to describe the reduction of GO according to reaction schemes (3) and (4). This occurs with a carbon loss by releasing $\mathrm{CO}_{2}$ originating from $\mathrm{COO}^{-}$and $\mathrm{COOH}$ respectively (3), and without carbon loss when the epoxy group (two $\mathrm{OH}$ groups) is removed (4).

$$
\begin{gathered}
\mathrm{GO}+\mathrm{e}_{(\mathrm{aq})}{ }^{-}+\mathrm{H}^{+} \rightarrow \mathrm{rGO}+\mathrm{H}_{2} \mathrm{O}+\mathrm{CO}_{2} \\
\mathrm{GO}+\mathrm{e}_{(\mathrm{aq})}{ }^{-}+\mathrm{H}^{+} \rightarrow \mathrm{rGO}+\mathrm{H}_{2} \mathrm{O}
\end{gathered}
$$

\section{Discussion}

The observed rate constants for all three GO samples were only differing by a factor of less than 2.5. Thus the determined rate constant for the reduction of GO by solvated electrons in water is between $1 \times 10^{7}$ and $3 \times 10^{7} \mathrm{ml} \mathrm{mg}^{-1} \mathrm{~s}^{-1}$. Our experimental data can be related to a lifetime of the solvated electron of about $35 \mathrm{~ns}$ for the conditions applied in ref. 1 and therefore deviates by roughly four orders of magnitude from the reported one. ${ }^{1}$

However, the results reported by Gengler et al. were obtained by laser photolysis, where $\mathrm{e}_{(\mathrm{aq})}{ }^{-}$was supposed to orig-
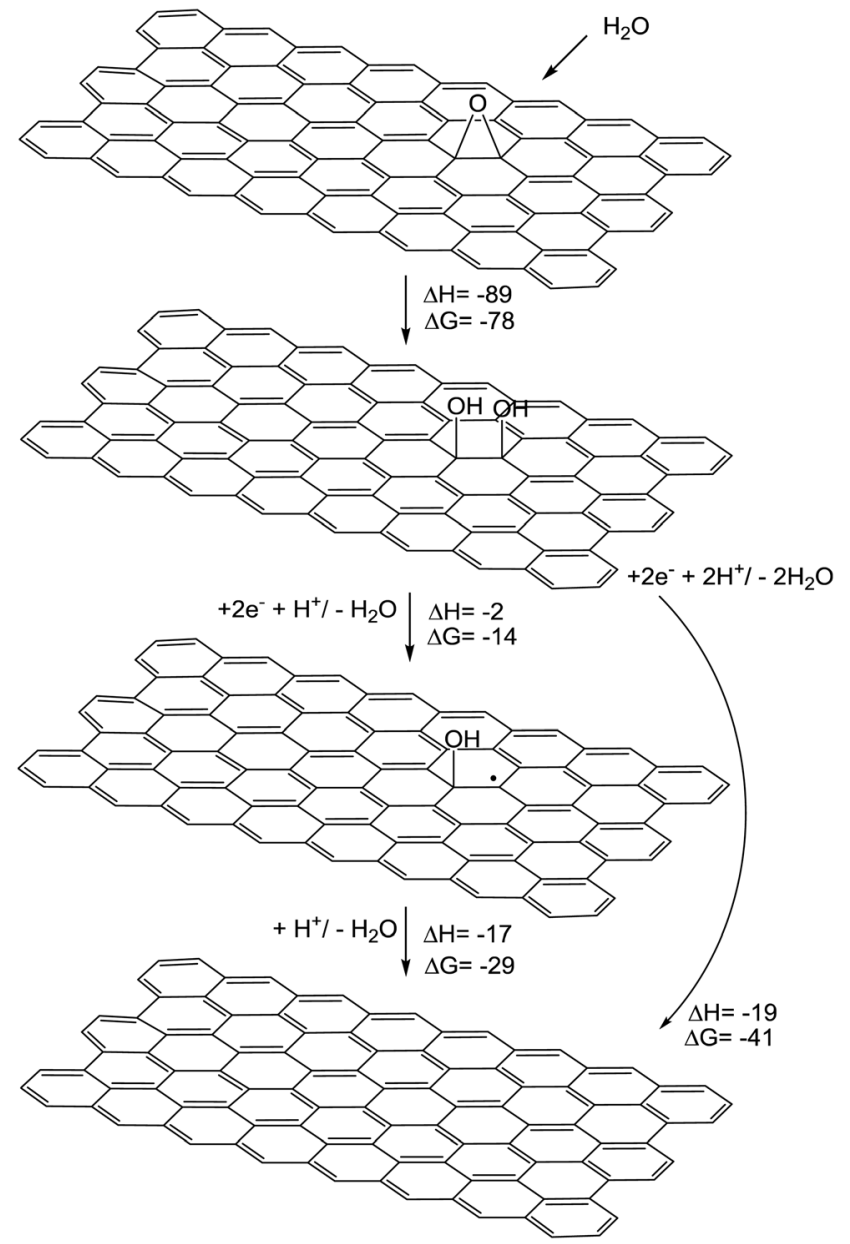

Fig. 3 Calculated energetics of the possible reaction pathways of $\mathrm{OH}$ removal after the interaction of the $\mathrm{GO}$ with $\left.\mathrm{e}_{(\mathrm{aq})}\right)^{-} . \Delta H$ and $\Delta G$ are given in $\mathrm{kcal} \mathrm{mol}^{-1}$.

inate from a two photon absorption of water. But this two photon absorption is in strong competition with the one photon-excitation and ionization of GO. Please note that GO shows strong absorption at the used excitation wavelength $(266 \mathrm{~nm})$ and the concentrations employed. The absorption of ai-GO is shown in Fig. S5 in the ESI. $\uparrow$ A standard way to determine whether their observed transient originates from one or two photon absorption is by determining the photonicity of the observed process, i.e., plotting the excitation energy vs. the transient absorption. A two photon absorption results in a parabola shaped curve, whereas a linear behaviour is observed when the transient originates from the absorption of one photon. In fact we observed the latter, a linear relationship in our femtosecond transient absorption measurements, when varying the energy between 100 and $400 \mathrm{~nJ}$ per pulse (see Fig. S6 $\dagger$ ). This result supports our interpretation/picture that the transients in ref. 1 are not due to two photon absorption/ionization of water and subsequent $\mathrm{e}^{-}$production from water but absorption of UV-radiation by highly concentrated GO and the subsequent release of an electron from photoionized GO. 
Theoretical calculations were conducted to elucidate how the reduction, described by Gengler et al. (which has been shown experimentally and which we do not doubt), could occur without the formation of a solvated electron from the two photon absorption of water. Our quantum chemical calculations showed that $\mathrm{GO}$ is ionized from photoexcitation at $266 \mathrm{~nm}$, forming a $\mathrm{GO}^{+} / \mathrm{e}^{-}$ion pair. A large quantity of electrons are not able to escape into water forming solvated electrons and are recombining on a short time scale (below $1 \mathrm{~ns})^{26,27}$ This recombination may, e.g., be accompanied by $\mathrm{CO}_{2}$ formation, as described in reaction scheme (5) (see also Fig. S7 in the ESI $\dagger$ ).

$$
\mathrm{GO}^{+}+\mathrm{e}^{-} \rightarrow \mathrm{rGO}+\mathrm{CO}_{2}
$$

It should be noted that carboxylic acids play a minor role for ai-GO, as the concentration of defects is on average only $0.3 \%$. This is much different for commercial GOs. The defect concentration is usually unknown, but approximately between 5 and $10 \%$. Although XPS can give information about the amount of carbonyl/carboxyl functions, their concentration is difficult to determine reliably in a quantitative fashion. Therefore, the exact concentration of carboxylic acids is not known precisely and the major functional groups remain epoxy and hydroxyl groups. Nevertheless, we included the mechanism of $\mathrm{CO}_{2}$ formation from carboxylic acids (beyond $\mathrm{OH}$ and epoxy group reduction), because this mechanism certainly becomes relevant for highly defective GO.

In summary, the photochemical conversion of GO to rGO observed in ref. 1 is not related to the reaction of GO with solvated electrons originating from water as in our experiments, but most likely from the laser excitation of the GO. Such a behaviour is not unexpected and well reported in the literature for the laser photolysis of pure GO dispersions. ${ }^{28,29}$

It should be noted, that we do not question the overall photochemical reduction of graphene in ref. 1 , such as shown impressively via XPS, but just the molecular mechanism. Our recent experimental results may indicate that while the direct photochemical reduction is rather inefficient, in principle, it may, however, become more efficient for high photon densities possibly forming $\mathrm{H}$ atoms (from solvated electrons via reaction with $\mathrm{H}^{+}$at the low $\mathrm{pH}$ values typical for highly concentrated GO dispersions), which is indeed an efficient reduction agent.

\section{Conclusions}

Efficient techniques to reduce graphene oxide are interesting due to the multitude of potential applications of this carbon material. A very promising method is its photochemical reduction employing ultraviolet light. Unfortunately, the dynamics and mechanisms behind this reduction remain elusive. Recently, Gengler et al. found that the ultraviolet photo-reduction in solution proceeds on a picosecond timescale, as measured with ultrafast spectroscopy. They propose that the reaction is initiated with a femtosecond ultraviolet pulse that photoionizes the solvent, liberating solvated electrons, which cause the reduction. In an effort to understand this surprising result on a fundamental level we performed a series of transient kinetic experiments employing three different graphene oxides with $\mathrm{e}_{(\mathrm{aq})}{ }^{-}$generated in aqueous solutions. Our experimental data can be related to a lifetime of the solvated electron of about $35 \mathrm{~ns}$ for the conditions applied in ref. 1 , which deviates by roughly four orders of magnitude from the reported timescale of reaction of solvated electrons with GO dispersed in aqueous solution. We therefore conclude that the ultrafast transients observed by Gengler et al. are most likely not to be related to the reaction of GO with solvated electrons originating from water, as in our experiments here, but from the direct laser excitation of the GO. An alternative mechanism has been proposed here on the basis of model quantum mechanical calculations, as outlined above. Regarding the fate of the electron, most likely it migrates into the $\pi$-system of the GO once it has reacted with it. Another scenario is that a photoelectron from GO is directly released into the solvent and reacts on a longer timescale with another neutral graphene oxide flake. The latter may explain a spectrum close to that of a nearly solvated electron at intermediate times. It is clear that GO in the experiments of Gengler et al. is ultimately reduced, the questions here is how and on which timescale. A further explanation for the ultrafast observations by Gengler et al. ${ }^{1}$ might also relate to trapped electrons formed upon photoexcitation as observed by Kaniyankandy et al. ${ }^{30}$

\section{Acknowledgements}

AK and BA gratefully acknowledge funding from the Deutsche Forschungsgemeinschaft (DFG) via grant KA 3491/2-1. We like to thank Prof. Dirk M. Guldi (Friedrich-Alexander-Universität Erlangen-Nürnberg) for his continuous support during the photochemical measurements. SE acknowledges the DFG via Grant No. EI 938/3-1 for funding and Prof. Dr Andreas Hirsch for his support at FAU Erlangen-Nürnberg. This work is also supported by the Cluster of Excellence 'Engineering of Advanced Materials (EAM)' and SFB953 funded by the DFG.

\section{References}

1 R. Y. N. Gengler, D. S. Badali, D. Zhang, K. Dimos, K. Spyrous, D. Gournis and R. J. D. Miller, Nat. Commun., 2013, 4, 2560.

2 D. M. Guldi and V. Sgobba, Chem. Commun., 2011, 47, 606.

3 A. Ciesielski and P. Samori, Chem. Soc. Rev., 2014, 43, 381.

4 S. Eigler and A. Hirsch, Angew. Chem., Int. Ed., 2014, 53, 7720 .

5 S. Pei and H.-M. Cheng, Carbon, 2012, 50, 3210 and references herein.

6 C. K. Chua and M. Pumera, Chem. Soc. Rev., 2014, 43, 291 and references herein. 
7 R. Flyunt, W. Knolle, A. Kahnt, A. Prager, A. Lotnyk, J. Malig, D. M. Guldi and B. Abel, Int. J. Radiat. Biol., 2014, 90, 468.

8 R. Flyunt, W. Knolle, A. Kahnt, S. Eigler, A. Lotnyk, T. Häupl, A. Prager, D. M. Guldi and B. Abel, Am. J. Nano Res. Appl., 2014, 2 , 9.

9 S. Eigler, Phys. Chem. Chem. Phys., 2014, 16, 19832.

10 S. Mao, H. Pu and J. Chen, RSC Adv., 2012, 2, 2643.

11 G. Williams and P. V. Kamat, Langmuir, 2009, 25, 1386.

12 L. J. Cote, R. Cruz-Silva and J. Huang, J. Am. Chem. Soc., 2009, 131, 11027.

13 Y. Matsumoto, M. Koinuma, S. Y. Kim, Y. Watanabe, T. Taniguchi, K. Hatakeyama, H. Tateishi and S. Ida, ACS Appl. Mater. Interfaces, 2010, 2, 3461.

14 I. V. Lightcap, S. Murphy, T. Schumer and P. V. Kamat, Phys. Chem. Lett., 2012, 3, 1453.

15 D. A. Sokolov, C. M. Rauleau, D. B. Grohegan and T. M. Orlando, Carbon, 2013, 53, 81.

16 S. Eigler, M. Enzelberger-Heim, S. Grimm, P. Hofmann, W. Kroener, A. Geworski, C. Dotzer, M. Röckert, J. Xiao, C. Papp, O. Lytken, H.-P. Steinrück, P. Müller and A. Hirsch, Adv. Mater., 2013, 25, 3583.

17 E. M. Fielden, Chemical Dosimetry of pulsed Electron and X-Ray Sources in the 1-20 MeV Range, in The Study of Fast Processes and Transient Species by Electron Pulse Radiolysis, ed. J. H. Baxendale and F. Busi, NATO Advances Study
Institutes Series C. Mathematical and Physical Sciences, 1982, vol. 86.

18 A. D. Becke, J. Chem. Phys., 1993, 98, 5648.

19 C. T. Lee, W. T. Yang and R. G. Parr, Phys. Rev. B: Condens. Matter, 1988, 37, 785.

20 Jaguar, version 8.3, Schrodinger, Inc., New York, NY, 2014.

21 D. J. Tannor, B. Marten, R. Murphy, R. A. Friesner, D. Sitkoff, A. Nicholls, B. Honig, M. Ringnalda and W. A. Goddard, J. Am. Chem. Soc., 1994, 116, 11875.

22 J. H. Baxendale and M. A. J. Rodgers, Chem. Soc. Rev., 1978, 7, 235.

23 G. V. Buxton, C. L. Greenstock, W. P. Helman and A. B. J. Ross, J. Phys. Chem. Ref. Data, 1988, 17, 513.

24 F.-Y. Jon and G. R. Freeman, J. Phys. Chem., 1977, 81, 909.

25 W. Braun, J. T. Herron and D. K. Kahaner, Int. J. Chem. Kinet., 1988, 20, 51.

26 D. Kozawa, X. Thu, Y. Miyauchi, S. Mouri, M. Ichida, H. Su and K. Matsuda, J. Phys. Chem. Lett., 2014, 5, 1754.

27 J. Shang, L. Ma, J. Li, W. Ai, T. Yu and G. G. Gurzadyan, J. Phys. D: Appl. Phys., 2014, 47, 094008.

28 L. Huang, Y. Liu, L.-C. Ji, Y.-Q. Xie, T. Wang and W.-Z. Shi, Carbon, 2011, 49, 2431.

29 R. Trusovas, K. Ratautas, G. Raciukaitis, J. Barkauskas, I. Stankeviciene, G. Niaura and R. Mazeikiene, Carbon, 2013, 52, 574.

30 S. Kaniyankandy, S. N. Achary, S. Rawalekar and H. N. Ghosh, J. Phys. Chem. C, 2011, 115, 19110. 